Freiburg

\title{
DIE OSKISCHE WEIHUNG AN FATUUS VE. 183
}

Die Inschrift Ve. 183 aus Civita di Tricarico (MT) gehört zu den oskischen Texten, die beim bisherigen Stand der Forschung als allenfalls halb verständlich gelten können. Die jüngste Gesamtinterpretation, die E. Vetters von $1953^{1}$, läßt drei der 12 Wörter unübersetzt und erklärt das Verständnis eines vierten als unklar. V. Pisani ${ }^{2}$, der auf Vetter fußt, läßt ebenfalls drei Wörter unübersetzt, darunter ein anderes als Vetter, und weicht an zwei weiteren Stellen von Vetter ab. Ein Gesamtverständnis gelingt ihm ebensowenig wie diesem. G. Bottiglioni ${ }^{3}$ schließlich referiert, mit Hinweis auf Vetters Vorbehalte, die Deutung Ribezzos ${ }^{4}$, die allein wegen der vielen angenommenen Abkürzungen verfehlt sein muB. Die hier vorgelegte Interpretation kann auch nicht alle Probleme des Textes lösen; doch hoffe ich, sowohl in den Details weiter als auch dem Gesamtverständnis näher zu kommen.

Ausgangspunkt ist eine Neulesung. Bei einer Autopsie am 27.3.1974 im Museo Provinciale von Potenza ${ }^{5}$ ergab sich, daß das dritte Wort des Textes, das auf den zweigliedrigen Personennamen $\kappa \lambda o F \alpha \tau \sigma \gamma \alpha v \kappa \imath \varepsilon \sigma$ folgt, mit $f \alpha[$ beginnt und nicht mit $\sigma \alpha \kappa$ [, wie Ribezzo gelesen hatte. Ribezzos Lesung war ganz offensichtlich durch das Bestreben geleitet, die Buchstabenreste zu der Wortform $\sigma \alpha \kappa[\rho \alpha \kappa \lambda \alpha(\mu)]$ zu ergänzen, wofür allerdings, wie schon Vetter bemerkte, die Lücke auf dem Stein nicht groß genug ist; auch wäre die Unterdriickung des auslautenden Nasals in griechisch geschriebenen oskischen Texten ohne Parallele. Das $>f<$ des griechisch-oskischen Alphabets kann dem vierstrichigen Sigma sehr ähnlich sein, sich von diesem nur durch das Fehlen der untersten Querhasta unterscheiden. Hier aber findet sich nicht nur keine Spur einer unteren Querhasta; vielmehr ist auch die obere Querhasta nicht waagrecht wie bei den anderen Sigmas des Textes (das der letzten Zeile ausgenommen), sondern nach links unten geneigt wie bei den anderen $>f<$. Unmittelbar hinter dem $\alpha$ ist kein Buchstabenrest zu erkennen. Die Lesung $f \alpha[$ statt $\sigma \alpha K[$ ist sicher.

Die Ergänzung von $f \alpha[$ ergibt sich aus der anderen mit dieser Buchstabenfolge beginnenden Wortform des Textes, aus $f \alpha \tau o F \varepsilon$ Z.4. Diese Form kann nicht Lokativ

1 E. Vetter, Handbuch der italischen Dialekte I, Heidelberg 1953, pp. 120-122 mit weiterer Literatur.

2 V. Pisani, Le lingue dell'Italia antica oltre il latino, Torino $1964^{2}$, p. $50 \mathrm{~s}$.

3 G. Bottiglioni, Manuale dei dialetti italici, Bologna 1954, p. 249 s.

4 Fr. Ribezzo, RIGI 8, 1924, pp. 89-92 (und vorher Neapolis 1, 1913/4, pp. 389 s.).

5 Durchgeführt zusammen mit W. Pfaffel, auf einer von der DFG geförderten Reise zur Kontrolle der Lesungen oskischer Inschriften; der DFG sei auch an dieser Stelle für die Förderung gedankt. 
Singular (Vetter) oder Dativ Singular (Pisani) eines $u$-Stammes sein, da der Ausgang dieser Formen auf der Inschrift $>F \varepsilon l<$ (oder $>F-\varepsilon V<$ ) bzw. $>F \eta l<$ geschrieben sein müßte, wie $>f \alpha \kappa \varepsilon l \tau<$ für [fakit] und $>\kappa \lambda o F \alpha \tau \eta \imath \sigma<$ für -[teis ] zeigen. Der andere oskische Beleg des Wortes, der Genetiv fatuveís (Ve. 165), gehört zu einem oStamm $^{6}$, und ein $o$-Stamm ist auch die offenkundige lateinische Entsprechung, der Göttername Fatuus. Die Form eines $o$-Stammes ist schließlich auch $f \alpha \tau o F \varepsilon-$ was noch niemand gesehen zu haben scheint -, und zwar der Vokativ, der auch syntaktisch und inhaltlich paßt, wie gleich zu zeigen ist. Nach dem anschließenden, noch in der Lücke beginnenden adjektivischen Epitheton $\delta] \iota^{2} O F t o l$ (so richtig Vetter) ist der Göttername zum Dativ $f \alpha[\tau o F o l$ zu ergänzen. Für die Buchstabenfolge $>\tau o F o t \delta<$ ist in der Lücke ausreichend Platz, da $o$ als Punkt geschrieben ist. Ein Göttername im Dativ hinter dem Namen des Dedikanten im Nominativ ist der übliche Anfang privater oskischer Weihinschriften: stenis. kalaviis. $g^{2}$ anagtiai. díviiai ${ }^{3}$... Ve. 140 (Aesernia), siviiú magiú ${ }^{2}$ mefit(eí) Ve. 162 (Aeclanum), $[\sigma \tau] \alpha \tau[\imath] \sigma[----\imath] \varepsilon \sigma \sigma o v F \eta \imath$ [---(-

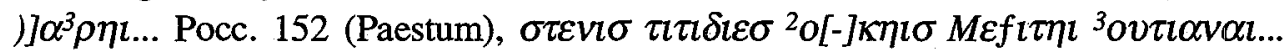
Pocc. 164 (Rossano di Vaglio), ].staíiiús ${ }^{2}$-?-Jínnianúi ${ }^{3}$... Pocc. 33 (Vastogirardi), maras.staíis.banttieí[s] ${ }^{2}$ lúvkis.dekitis.marah[eís] ${ }^{3}$ vikturraí ... Pocc. 16 (Pietrabbondante) $)^{7}$.

Die Belege Vok. $f \alpha \tau o F \varepsilon$ und Dat. $f \alpha[\tau o F o t]$ dürfen (neben fatuveís Ve. 167) in Lejeunes Liste der Theonyme auf oskischen Inschriften (REA 74, 1972, p. 13) aufgenommen werden. Die lautgesetzliche Form des Stammes vor vokalisch anlautender Endung müßte nach der gemeinsabellischen Synkope fatuno- oder, mit Stützvokal wie in praefucus etc., fatuuno- wie in fatuveís Ve. $167^{8}$ lauten, die Formen also * $>f \alpha \tau F \varepsilon$, $f \alpha \tau F o l<$ bzw. * $>f \alpha \tau o v F \varepsilon, f \alpha \tau o v F o l<$. Die Form $f \alpha \tau o F \varepsilon$ (dann wohl auch $f \alpha[\tau o-$ FolI) sind nach dem Nominativ ${ }^{*} f \alpha \tau o F \sigma$ gebildet, wo die Endsilbensynkope eine Konsonantengruppe geschaffen hat, die wiederum die Binnensilbensynkope verhinderte.

Damit ist der erste Satz des Textes bis auf eine Wortform voll verständlich. Er lautet, mit der traditionellen Interpunktion, mit der Konjektur $\alpha f \alpha \kappa \varepsilon l \tau$ für $\alpha f \lambda \kappa \varepsilon \imath \tau^{9}$ und mit der neuen Ergänzung

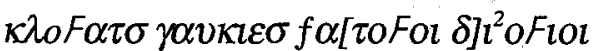

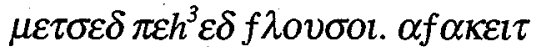

6 Worauf Vetter selbst verweist, ohne Konsequenzen zu ziehen. - Die theoretische Möglichkeit eines $i$ Stamms kann hier, wie das folgende zeigt, ignoriert werden.

7 Ausnahme: $\mu \varepsilon f \imath \tau \varepsilon \imath \mu \alpha \rho \alpha \sigma^{2} \sigma \tau \alpha \lambda \lambda \imath \varepsilon \sigma$... Pocc. 182 (Rossano di Vaglio).

8 Im Hirpinergebiet erfolgte zwischen $t$ und dem Stützvokal $u$ kein Einschub eines $i$ (was M. Lejeune, REA 72, 1970, p. 297, als Ausnahme vermerkt), so wenig wie in Bantia (eituam Ve. 2, 13 etc.), aber anders als in Kampanien (eítiuvam Ve. 11) und in Samnium (eitiv(ad) Ve. 142).

9 Dazu zuletzt A.L. Prosdocimi, Lingue e dialetti dell'Italia antica, ed. A.L. Prosdocimi, Roma 1978, p: 1060. 
und er bedeutet: 'Clovatus Gavicius weiht ${ }^{10}$ dem Fatuus Iovius recht ${ }^{11}$ und ehrerbietig

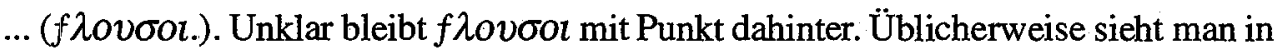
letzterem einen Worttrennungspunkt und in der Form einen Dativ eines $o$-Stammes * flōso- = lat. Flōrus. Doch welche Funktion hätte dieser? Der Adressat der Weihung ist Fatuus Iovius, und auch wer die hier vorgeschlagene Ergänzung nicht annehmen wollte, müßte sich damit auseinandersetzen, daß lat. Flōrus ein Personenname ișt (der Göttername ist Flōra = osk. Flōzā- in Dat. fluusaí Ve. 21; 147 A 24) und daß eine Person sich schlecht als Adressat einer Weihung eignet (lat. facere und seine sabellischen Entsprechungen drücken in vergleichbaren Zusammenhängen stets Kulthandlungen aus, hierin die alte Bedeutung der Wurzel $* d^{h} e h^{-}$- fortsetzend ${ }^{12}$ ); auch bliebe die Sperrung der beiden Dative $\delta 7 \imath \circ F_{l o l}$ und $f \lambda \circ v \sigma o \imath$ merkwürdig. Eine Interpretation von $f \lambda o v \sigma o t$ als Dativus commodi ('für Florus') stößt sich wiederum am Dativ des Adressaten; man würde für den Interessenten einen Präpositionalausdruck mit pru oder úp erwarten.

Nun ist es freilich durchaus nicht so selbstverstänldich, wie uns die Handbücher glauben machen, daß der Punkt in der Inschrift zwei verschiedene Funktionen hat, nämlich achtmal ein $>0<\mathrm{zu}$ bezeichnen und zweimal die Worttrennung, die andere acht Male unbezeichnet blieb. Die Punkte einheitlich als Worttrenner zu lesen, ist so absurd, daß es noch niemand vorgeschlagen hat (und jeder, der es versucht, wird dies verstehen). Die Alternative, eine konsequente Interpretation des Punktes als $>0<$, führt an der hier interessierenden Stelle (zur anderen später) zu einer Wortform $f \lambda o v-$ бoıo. Diese könnte der Nominativ-Akkusativ Plural eines neutralen Substantivs oder Adjektivs sein wie etwa $\sigma \alpha \kappa o \rho o$ sacra' Ve. 196 (Messina) oder $\sigma \tau \alpha \beta \alpha \lambda \alpha v o$ erigenda' Pocc. $175^{13}$ (Rossano di Vaglio), und damit das Akkusativobjekt zu $\alpha f \alpha \kappa \varepsilon \imath \tau$

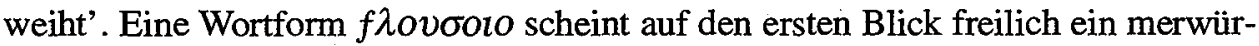
diges Gebilde zu sein; denn intervokalisches $i$ ist schon uritalisch geschwunden ${ }^{14}$, und von den Bedingungen für die Entstehung eines $-i j-$ ist keine gegeben: Lok. Sing. auf $-V i+$ Suffix -io- (osk. púmpaiians, umbr. pernaiaf; der Lok. Sing. der o-Stämme endet auf - ei , der der Konsonantenstämme auf $-i)$; urital. gi - und ursabell. - hi - (o.

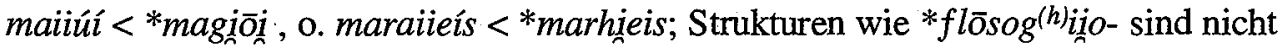
motivierbar).

10 Nicht Perfekt (Pisani), sondern Präsens (Vetter; M. Lejeune, REA 72, 1970, p. 291; zuerst Fr. Ribezzo, RIGI 8, 1924, pp. 90 s.; dann C.D. Buck, A grammar of Oscan and Umbrian, Boston $1929^{2}=$ Hildesheim 1974).

11 Wohl für * $\mu \varepsilon \tau \sigma \tau \varepsilon \delta<*$ medos-tẹd (Vetter).

12 J. Untermann, Indogermanica et Italica = Festschrift H. Rix, Innsbruck 1993, p. 466.

13 Im gleichen Text mit jüngerer Orthographie $(\omega) \pi \sigma \alpha v \omega$ facienda' und $\alpha t \zeta v \imath \omega$ ahenea', sowie mit Mischorthographie $\sigma \varepsilon \gamma \nu v \omega$ signa' (der anaptyklische Vokal ist mit seinem Muster lautlich identisch), $\mathrm{cf}$. Lejeune, REA 74 (1972), p. 10.

14 M. Leumann, Lat. Grammatik (Neuausgabe) 1977, p. 126; F. Sommer-R. Pfister, Handbuch der lat. Lautund Formenlehre I, Lautlehre, Heidelberg 1977, pp. 173 ss., G. Meiser, Lautgeschichte der umbrischen Sprache, Innsbruck 1986, p. 38. 
Es gibt aber doch einen - zugegeben schmalen, aber doch gangbaren - Weg, eine Form $f \lambda o v \sigma o t o$ [flōsaiiåa] zu verstehen. Er wird durch die Form búvaianúd Ve. 150 (Pietrabbondante) = lat. Boviāno angedeutet, in der in die Lautgruppe $V_{1, u i n} V_{2}<$ $V_{1} u i V_{n} V_{2}$ ein anaptyktischer Vokal $V_{2}$ eingeschoben ist. Auf den vorliegenden Fall übertragen, ergäbe sich eine Entwicklung $V_{I} s i{ }_{n} V_{2}>V_{I} i V_{2}>V_{2} z V_{2} i V_{2}$, konkret ${ }^{*}$ flọsijiō $>$

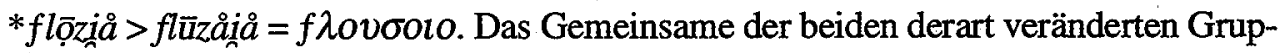
pen wäre die intervokalische Folge von stimmhaftem, nicht-nasalem oder -liquiden Dauerlaut $+i{ }^{i}$. Wie im Fall búvaianúd ist dabei das $i$ erst durch die Anaptyxe $( \pm 300$ v.Chr.) zwischen Vokale geraten. Eine Morphostruktur urosk. (oder ursabell.) ${ }^{*} f l \bar{o} s-$ iijo- (daraus mit Synkope *flọzz-io- und mit Anaptyxe flūsoio-) ist aber als -iīo-Zugehörigkeitsbildung zu *flọs- Blume, Blüte' alles andere als ungewöhnlich. Altererbte und junge Bildungen mit Suffix -iiio- > -io- sind im Oskischen wie überhaupt im Sabellischen gerade im weiteren Bereich des religiösen Wortschatzes nicht selten: osk. fiísíaís Ve. $86+$ (Capua) = lat. fëriae $<{ }^{*}$ feesiiāa; osk. (húrtín) kerriiín + Ve 147 (in horto) Cereali' < *kersēieir; osk. Mamerttiaís Ve 86 + (Capua) <-mert(i)ina-; weiter umbr. gomia kumiaf trächtig' TIg. VI a 58. I a 7 * gom(o)-iiāa- und umbr. feliuf filiu säugend' T I a 14. VI b 3, schließlich sabell. *boun-(i)i̇o- zum Rind gehörig', das Grundwort von búvaianúd. Das Grundwort, die Entsprechung von lat. flōs, ist im Sabellischen zwar nicht direkt, aber in der dem lateinischen Götternamen Flōra entsprechenden Weiterbildung belegt: osk. Fluusaí (Dat. Sg.) Ve. 25 (Pompei) und 147 (Agnone).

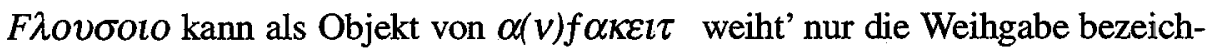
nen, möglicherweise eine öfter dargebrachte Weihgabe, wenn man das Präsens $\alpha f \alpha \kappa \varepsilon l \tau$ ernstnehmen könnte. Als Pluralform kann $f \lambda o v \sigma o t o$ auch nicht den beschrifteten Gegenstand meinen, einen vorne profilierten Steinblock von $70 \mathrm{~cm}$ Höhe, $52 \mathrm{~cm}$ Breite und $40 \mathrm{~cm}$ Tiefe. Dieser ist vielmehr ganz offensichtlich ein kleiner Altar, auf dem die Weihgaben dargebracht wurden. Hatte Fatuus in Lukanien die gleiche Rolle, die Plinius (nat. 27, 107) ihm für Picenum zuschreibt, nämlich die Wöchnerinnen zu schädigen ${ }^{15}$, dann könnten die Weihgaben den Zweck gehabt haben, den Gott zu besänftigen und von seinem Vorhaben abzubringen. Nun war aber osk. flọssiokaum das allgemeine Wort für Weihgabe'; er muß eine speziellere, mit der des Basisworts flọs- zusammenhängende Bedeutung gehabt haben, wenn auch nicht gerade mit der eigentlichen, weil dafür wieder das eigentliche Wort, etwa das für Kranz' gewählt worden wäre und nicht eine Umschreibung zur Blume/Blüte gehörig'. Dies ist jedoch gut vorstellbar, wenn osk. ${ }^{*} f l o ̄ s-$ ebenso wie lat. flōs und griech. $\ddot{\alpha} v \vartheta o \varsigma$ auch im Sinn das Beste von' gebraucht werden konnte (lat. flos nobilitatis, vini, olei,

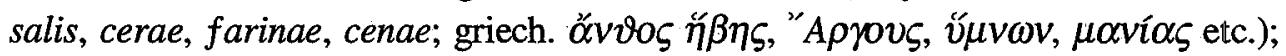
dann könnte flōs $(i)$ ilo- zum Besten seiner Art gehörig' geheißen haben. Doch weiter soll der Sprachwissenschaftler nicht in das Revier des Religionswissenschaftlers vor-

K. Latte, Röm. Religionsgeschichte, München 1960, p. $84^{2}$. 


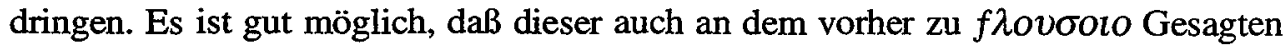
Korrekturen anzubringen hat.

Abgesehen davon, daß die spezifische Bedeutung von f $\lambda$ ovooto unsicher' bleibt, da sie nur sprachvergleichend erschlossen, nicht aber auch sachvergleichend bestätigt ist, kann man den ersten Satz des Textes nun als lexikalisch, syntaktisch und inhaltlich verständlich ansehen.

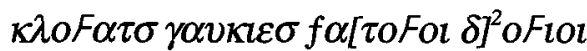

$$
\begin{aligned}
& \mu \varepsilon \tau \sigma \varepsilon \delta \pi \varepsilon h^{3} \varepsilon \delta \text { f } \lambda \text { ov } \sigma o l o \alpha f \alpha \kappa \varepsilon \imath \tau
\end{aligned}
$$

Clovatus Gavicius weiht dem Fatuus Iovius recht und ehrerbietig die (?) Erstlingsgaben'

Syntaktisch klar ist auch der zweite Satz; da aber über die Bedeutung des letzten Wortes derzeit keine auch nur einigermaßen konkretisierbare Vermutung möglich ist, ist auch der Gesamtinhalt nur sehr generell zu verstehen. Der Text lautet, wenn man auch hier den scheinbaren Worttrennungspunkt als $>0<$ liest

\section{${ }^{4} \alpha v \tau \iota$ o $f \alpha \tau o \digamma \varepsilon \kappa \lambda o^{5} F \alpha \tau \eta \iota \sigma \pi \lambda \alpha \mu \varepsilon \tau o \delta$}

und ist zu verstehen als: Aber, o Fatuus, .. [Imperativ].. (das) des Clovatus'. Er enthält also die Bitte, die der Dedikant mit seiner Weihung verbindet, im Imperativ II wie bei Bitten an die Gottheit üblich. Die bekannte Adversativkonjunktion $\alpha v \tau \iota$ setzt sie von der Konstatierung der Weihung ab. Der Vokativ $f \alpha \tau o F \varepsilon$ wurde schon oben besprochen. Die Annahme, daß davor die aus dem Lateinischen und Griechischen bekannte Anredepartikel $o[\bar{o}]$ steht und daß sich diese dem sabellischen Lautwandel zu $\bar{o}$ (bzw. hier $>\bar{u}$ ) entzogen hat, ist unproblematisch. Da die Bedeutung des Prädikatsverbs $\pi \lambda \alpha \mu \varepsilon \tau \circ \delta$ (einstweilen) unbekannt ist, muß offen bleiben, ob der Genetiv $\kappa \lambda o_{-}$ $F \alpha \tau \eta \imath \sigma$ ein Objektsgenetiv ist, wie er etwa neben lat. miseret steht, oder elliptisch für (das Haus) des Clovatus' (Typ griech. $\varepsilon i \zeta^{\text {"A } A l \delta o v) ; ~ j e d e n f a l l s ~ b e z i e h t ~ s i c h ~ d i e ~ B i t t e ~}$ auf den Dedikanten selbst oder auf seinen näheren Umkreis. Nach der oben erwähnten Funktion des Fatuus als Wöchnerinnenschreck wären Bedeutungen des Typs erbarme dich des C.' oder verschone (das Haus) des C.' denkbar. Eine Klärung könnte ein weiterer Beleg des Verbums bringen, eine Andeutung wenigstens die Etymologie, wenn diese gefunden ist.

\section{Povzetek \\ POSVETITEV FATUUSU VE 183 (V OSKUSŠČINI)}

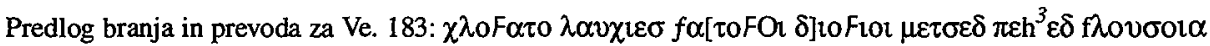
$\alpha f \alpha \chi e \iota \tau{ }^{4} \alpha v \pi \tau$ o $f \propto \tau 0 F \varepsilon \chi \lambda 0^{5} F \alpha \tau \eta 1 \sigma \pi \lambda \alpha \mu \varepsilon \tau o \delta$ 'Clovatus Gavicius posveča Fat[uusu] Ioviousu prvino (= prvo od letnega pridelka) pravilno in spoštljivo. Toda, o Fatuus, $\pi \lambda \alpha \mu \varepsilon \tau o \delta$ (velelnik) Clovatusa'. Novosti tega

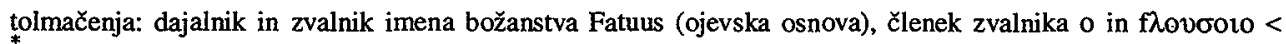
flọs(i)ia prvina. 